\title{
The Metod of Control of Influence of Temperature on Processes of Oxidation and Thermal Destruction of Full Synthetic Motor Oil ALPHA'S 5W-40 SN
}

\author{
Evgeniy A. Ermilov*, \\ Yuriy N. Bezborodov and Boleslav I. Kowalski \\ Siberian Federal University \\ 79 Svobodny, Krasnoyarsk, 660041, Russia
}

Received 06.10.2019, received in revised form 28.10.2019, accepted 05.12.2019

The results of the study of influence of the processes of oxidation and thermal destruction on the optical properties, the kinematic viscosity, volatility and anti-wear properties of full synthetic motor oil are presented. It was found, that the processes of thermal destruction have reduced the rate of change of optical density, volatility, kinematic viscosity and anti-wear properties, however the processes of thermal destruction have dominated at the processes of oxidation, when optical density is more 0,4.

Keywords: optical density, volatility, coefficient of ratable viscosity, index of thermooxidation stability and thermal withstandability, increment velocity of processes of oxidation and thermal destruction, index of anti-wear properties.

Citation: Ermilov E.A., Bezborodov Yu.N., Kowalski B.I. The metod of control of influence of temperature on processes of oxidation and thermal destruction of full synthetic motor oil ALPHA'S 5W-40 SN, J. Sib. Fed. Univ. Eng. technol., 2019, 12(8), 972-979. DOI: 10.17516/1999-494X-0197.

(C) Siberian Federal University. All rights reserved

This work is licensed under a Creative Commons Attribution-NonCommercial 4.0 International License (CC BY-NC 4.0).

* Corresponding author E-mail address: evermilov@mail.ru 


\title{
Метод контроля влияния температуры
}

\author{
на процессы окисления и температурной деструкции \\ синтетического моторного масла ALPHA'S 5W-40 SN
}

\author{
Е.А. Ермилов, Ю.Н. Безбородов, Б.И. Ковальский \\ Сибирский федеральный университет \\ Россия, 660041, Красноярск, пр. Свободный, 79
}

\begin{abstract}
Представлены результаты исследования влияния процессов окисления и температурной деструкиии на оптические свойства, кинематическую вязкость, испаряемость и противочзносные свойства синтетического моторного масла. Установлено, что процессы температурной деструкиии замедляют скорость изменения оптической плотности, испаряемость и понижают кинематическую вязкость и противоизносные свойства, однако при оптической плотности больше 0,4 процессы температурной деструкции преобладают над процессами окисления.
\end{abstract}

Ключевые слова: оптическая плотность, испаряемость, коэффициент относительной вязкости, показатели термоокислительной стабильности и температурной деструкции, приращение скорости процессов окисления и температурной стойкости, показатели противоизносных свойств при окислении и температурной деструкции.

При эксплуатации двигателя внутреннего сгорания (ДВС) на поверхностях трения одновременно протекают процессы окисления, температурной деструкции и химические реакции металлов с их продуктами и присадками. Однако доминирующее влияние одного из процессов на физико-химические и противоизносные свойства масел изучены недостаточно. Решение этих вопросов позволит разработать мероприятия по повышению либо термоокислительной стабильности, либо температурной стойкости, что увеличит их ресурс и повысит надежность ДВС путем разработки новых присадок [1]. Поэтому целью настоящих исследований является определение доминирующего влияния продуктов окисления или температурной деструкции на изменение оптических свойств, испаряемости, кинематической вязкости и противоизносных свойств синтетического моторного масла ALPHA’S 5W-40 SN.

Методика предусматривала применение следующих средств контроля и испытания: прибора для оценки термоокислительной стабильности, прибора для оценки температурной стойкости, малообъемного вискозиметра, фотометра, трехшариковой машины трения, оптического микроскопа «Альтами МЕТ 1М» и электронных весов. Технические характеристики приборов приведены в работах [2-4].

Методика испытания синтетического моторного масла ALPHA'S 5W-40 SN на термоокислительную стабильность предусматривала два этапа [5]. На первом этапе пробу масла массой $(100 \pm 0,1)$ г заливали в стеклянный стакан прибора для оценки термоокислительной стабильности и термостатировали при температуре $180{ }^{\circ} \mathrm{C}$ с перемешиванием стеклянной мешалкой с частотой вращения 300 об/мин. После каждых 8 ч испытания стакан с окисленным маслом взвешивали для определения массы испарившегося масла, отбирали часть пробы (2 г) для прямого фотометрирования и определения оптической плотности D при толщине фотометрируемого слоя 2 мм:

$$
-973-
$$




$$
D=\lg \frac{300}{\Pi},
$$

где 300 - задаваемый ток фотометра при пустой кювете, мкА; П - показатель фотометра при фотометрировании масел, мкА.

Часть пробы окисленного масла (9 г) использовали для измерения кинематической вязкости. После измерения названных показателей окисленные масла сливали в стакан, который повторно взвешивали, и испытания продолжали до достижения оптической плотности D значений 0,5-0,6.

Второй этап заключался в оценке противоизносных свойств окисленных масел на трехшариковой машине трения со схемой «шар-цилиндр». Пробу окисленного масла массой 20 г отбирали в момент достижения оптической плотности $\mathrm{D}$ значений, приблизительно равных 0,1 ; 0,$2 ; 0,3 \ldots 0,6$, после чего пробу масла в стакане доливали товарным маслом до первоначальной массы $(100 \pm 0,1)$ г. Параметры трения составляли: нагрузка $10 \mathrm{H}$, линейная скорость вращения цилиндра $0,68 \mathrm{~m} / \mathrm{c}$, температура масла в объеме $80^{\circ} \mathrm{C}$, время испытания 1,5 ч. Противоизносные свойства оценивали по среднеарифметическому значению диаметра пятна износа на трех шарах с двух опытов [6-8].

Методика испытания температурной стойкости предусматривала два этапа. На первом этапе пробу масла массой $(100 \pm 0,1)$ г заливали в стеклянный стакан прибора для оценки температурной стойкости и термостатировали при температуре $180{ }^{\circ} \mathrm{C}$ без перемешивания при атмосферном давлении с конденсацией паров и отводом конденсата. После каждых 8 ч испытания стакан с термостатированным маслом взвешивали для определения массы испарившегося масла, отбирали часть пробы для прямого фотометрирования и определения оптической плотности D по формуле (1). Часть пробы окисленного масла использовали для измерения кинематической вязкости.

После измерения указанных показателей термостатированное масло с фотометра и вискозиметра сливали в стакан, который повторно взвешивали, и испытания продолжались до достижения оптической плотности D значений, равных 0,5-0,6.

Второй этап заключался в оценке влияния продуктов температурной деструкции на противоизносные свойства. Для этого масло испытывали по вышеописанной технологии с определением тех же показателей и дополнительно отбирали пробу масла массой 20 г для испытания на трехшариковой машине трения с теми же параметрами трения. Пробу термостатированного масла массой 20 г отбирали в момент достижения оптической плотности $\mathrm{D}$ значений, приблизительно равных 0,$1 ; 0,2 ; 0,3 \ldots 0,6$, а пробу масла в стакане прибора для оценки температурной стойкости доливали товарным маслом до первоначальной массы $(100 \pm 0,1)$ г.

\section{Результаты исследования и их обсуждения}

На рис. 1 представлены зависимости оптической плотности (а), испаряемости (б) и коэффициента относительной вязкости (в) от времени испытания исследуемого синтетического моторного масла при окислении (кривая 1) и температурной деструкции (кривая 2). Согласно данным (рис. $1 a$ ), в начальный период испытания в течение 16 ч интенсивность процессов окисления и температурной деструкции одинакова, дальнейшее увеличение времени испытания от 16 до 56 ч вызывает понижение интенсивности процессов температурной деструкции (кривая 2), что 
объясняется отсутствием перемешивания масла при термостатировании. Однако после 56 ч испытания интенсивность процессов температурной деструкции превышает процессы окисления. Так, значение оптической плотности $\mathrm{D}=0,5$ достигается за 79 ч при окислении и за 62 ч при температурной деструкции.

Испаряемость масла (рис. 1б) значительно уменьшается при процессах температурной деструкции. Так, за 72 ч испытания испаряемость составила при окислении 11 г (кривая 1), а при температурной деструкции 5,7 г (кривая 2). Это объясняется тем, что при температурной деструкции масло не перемешивалось и контакт с кислородом воздуха практически отсутствовал.

Изменение кинематической вязкости при термостатировании оценивалось коэффициентом относительной вязкости $K_{\mu}$, определяемым отношением
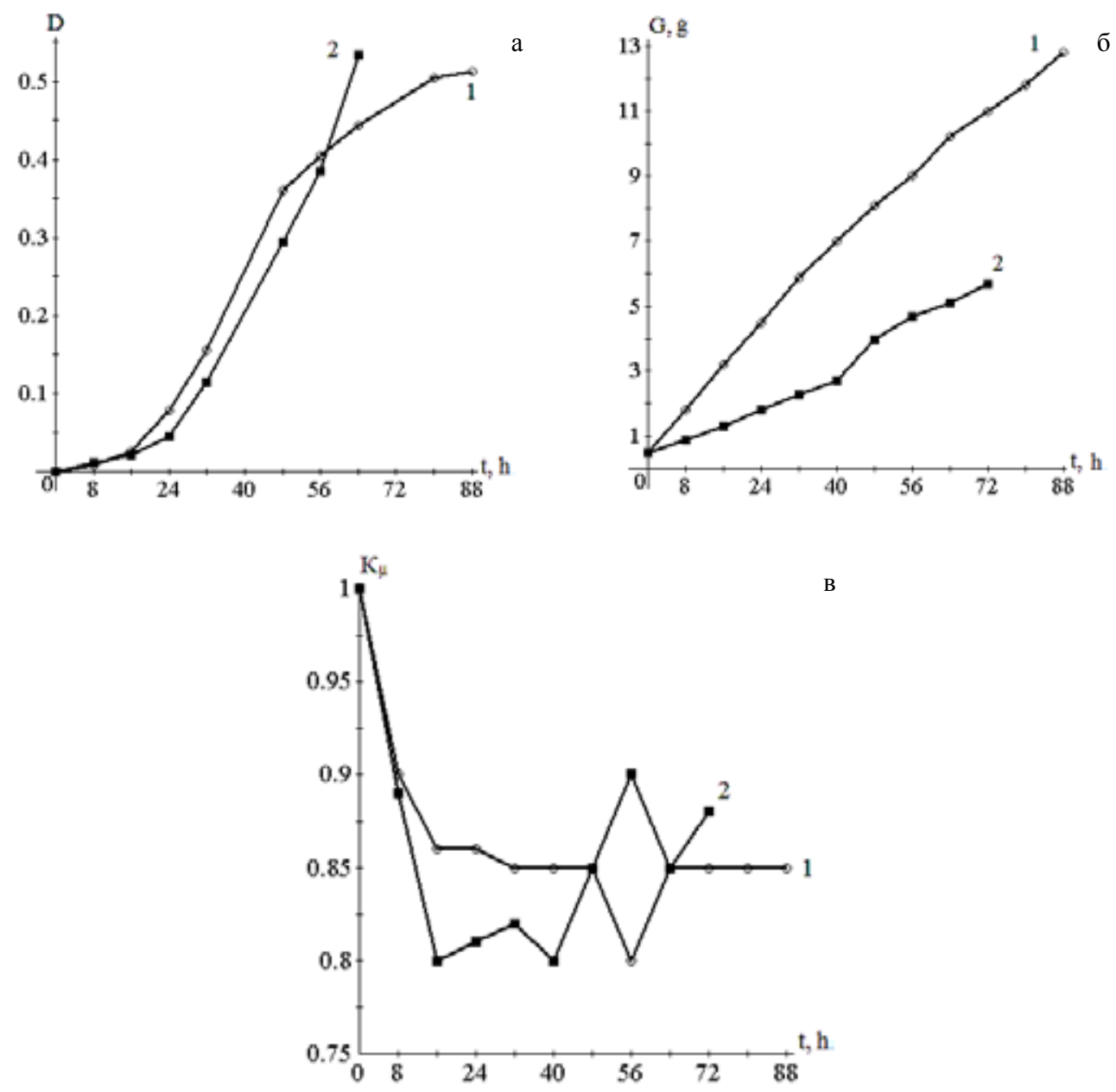

Рис. 1. Зависимости оптической плотности (а), испаряемости (б) и коэффициента относительной вязкости (в) от времени и температуры испытания $\left(180^{\circ} \mathrm{C}\right)$ синтетического моторного масла ALPHA'S $5 \mathrm{~W}-40$ SN: 1 - при окислении; 2 - при температурной деструкции

Fig. 1. Dependence of optical density (a), volatility (б) and relative viscosity coefficient (в) on time and temperature of testing $\left(180^{\circ} \mathrm{C}\right)$ of ALPHA'S $5 \mathrm{~W}-40 \mathrm{SN}$ synthetic motor oil: 1 - during oxidation; 2 - at temperature destruction 


$$
K_{\mu}=\frac{\mu_{T}}{\mu_{\text {ИCX }}}
$$

где $\mu_{\mathrm{T}}$ - вязкость термостатированного масла при окислении или температурной деструкции, $\mathrm{Mм}^{2} / \mathrm{c} ; \mu_{\text {исх }}$ - вязкость товарного масла, $\mathrm{Mм}^{2} / \mathrm{c}$.

Согласно данным (рис. 18), при окислении (кривая 1) и температурной деструкции (кривая 2) сохраняется общая тенденция уменьшения коэффициента $\mathrm{K}_{\mu}$; при термостатировании, однако продукты температурной деструкции понижают кинематическую вязкость более интенсивно. Так, после 40 ч испытания коэффициент $K_{\mu}$ при окислении уменьшился от 1 до 0,85, а при температурной деструкции - до 0,8 . Такое падение вязкости вызвано деструкцией вязкостной присадки и подтверждает различие в составе продуктов окисления и температурной деструкции, так как при окислении в масле образуются кислые продукты, которые отсутствуют при деструкии.

На рис. 2 представлены зависимости показателей Р термоокислительной стабильности (кривая 1) и температурной деструкции (кривая 2), определяемые выражением

$$
P=D+K_{G},
$$

где $\mathrm{K}_{\mathrm{G}}$ - коэффициент испаряемости

$$
K_{G}=\frac{m}{M},
$$

где $\mathrm{m}$ - масса испарившегося масла при окислении или температурной деструкции, г; $\mathrm{M}$ - масса пробы масла перед испытаниями за определенное время термостатированния, г.

Показатель Р характеризует количество тепловой энергии, поглощенной смазочным маслом при термостатировании и образовании определенной концентрации продуктов окисления или температурной деструкции и испарения. Согласно данным на рис. 2, в течение 60 ч про-

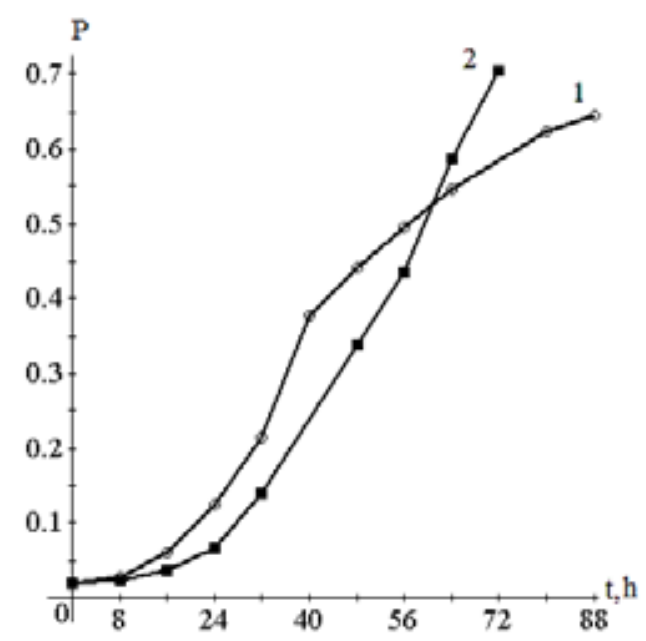

Рис. 2. Зависимости показателей термоокислительной стабильности (кривая 1) и температурной стойкости (кривая 2) от времени и температуры испытания $180{ }^{\circ} \mathrm{C}$ синтетического моторного масла ALPHA'S 5W$40 \mathrm{SN}$

Fig. 2. Dependences of thermal oxidative stability (curve 1) and temperature resistance (curve 2) on the time and temperature of testing $180^{\circ} \mathrm{C}$ of synthetic motor oil ALPHA'S 5W-40 SN

$$
-976-
$$


цессы температурной деструкции протекают с меньшей интенсивностью, чем процессы окисления (кривая 1).

Для сравнения интенсивности процессов окисления и температурной деструкции предложен параметр приращения скорости этих процессов $\Delta \mathrm{V}_{\mathrm{D}}$, определяемый выражением

$$
\Delta V_{D}=\left(D_{2}-D_{1}\right) / t
$$

На рис. 3 изображены зависимости приращений скорости процессов окисления и температурной деструкции за определенные отрезки времени испытания. Согласно данным, установлена общая тенденция увеличения приращений скоростей процессов окисления и температурной деструкции в начальный период термостатирования, однако интенсивность процессов окисления (кривая 1) выше, чем процессов температурной деструкции. Такое увеличение приращения скоростей вызвано ростом концентрации продуктов окисления и деструкции в масле.

Вторые участки зависимостей $\Delta \mathrm{V}_{\mathrm{D}}$ от времени испытания характеризуются значительными колебаниями приращения скоростей как процессов окисления, так и процессов температурной деструкции, что объясняется образованием продуктов этих процессов с большей энергоемкостью. Во время испытания исследуемого масла первичные продукты, образующиеся в начальный период, концентрация которых непрерывно росла, переходят в более энергоемкие, требующие больше тепловой энергии для их образования, а так как выделяемая тепловая энергия постоянна, то время образования энергоемких продуктов увеличивается, вызывая уменьшение приращения скорости процессов окисления и температурной деструкции. Причем переход первичных продуктов окисления в энергоемкие наступают после 40 ч испытания, а первичных продуктов деструкции в энергоемкие после - 48 ч.

Важным показателем эксплуатационных свойств моторных масел являются их противоизносные свойства, обеспечивающие надежность двигателей. В этой связи важно установить доминирующее влияние на противоизносные свойства продуктов окисления или температурной

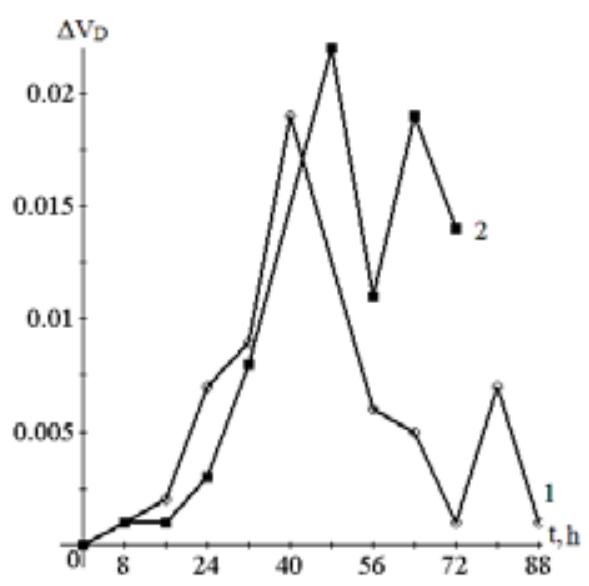

Рис. 3. Зависимости приращения скоростей окисления (кривая 1) и температурной деструкции (кривая 2) от времени и температуры испытания $180{ }^{\circ} \mathrm{C}$ синтетического моторного масла ALPHA'S 5W-40 SN

Fig. 3. Dependences of the increment of oxidation rates (curve 1) and temperature degradation (curve 2) on the time and temperature of testing $180{ }^{\circ} \mathrm{C}$ of ALPHA'S 5W-40 SN synthetic motor oil

$$
-977-
$$


деструкции. В данной работе предложен показатель противоизносных свойств $P_{A V}$, определяемый выражением

$$
P_{A V}=D / H,
$$

где И - среднеарифметическое значение диаметра пятна износа на трех шарах, мм.

Данный показатель характеризует концентрацию продуктов окисления или температурной деструкции на номинальной площади фрикционного контакта.

На рис. 4 представлены зависимости показателя противоизносных свойств от оптической плотности.

Установлено, что данные зависимости описываются линейными уравнениями

$$
\boldsymbol{P}_{A V}=\alpha \boldsymbol{D},
$$

где $\alpha$ - скорость изменения показателя $P_{A V}$.

Регрессионные уравнения зависимостей имеют вид для: процессов окисления (кривая 1) $P_{A V}=3,958 D$, процессов температурной деструкции $P_{A V}=3,402 D$.

Коэффициент корреляции составил 0,989 и 0,993 соответственно.

Согласно данным (рис. 4) противоизносные свойства при окислении превышают противоизносные свойства термостатированных масел в 1,2 раза, кроме того, при температурной деструкции моторного масла значения показателя $P_{A V}$ более стабильны.

\section{Выводы}

На основании проведенных исследований установлено:

1. Процессы температурной деструкции оказывают меньшее влияние на оптические свойства синтетического моторного масла ALPHA'S 5W-40 SN, уменьшают скорость испарения и увеличивают кинематическую вязкость.



Рис. 4. Зависимости показателя противоизносных свойств при окислении (кривая 1) и температурной деструкции (кривая 2) от оптической плотности и температуры испытания $180{ }^{\circ} \mathrm{C}$ синтетического моторного масла ALPHA'S 5W-40 SN

Fig. 4. Dependence of the indicator of antiwear properties during oxidation (curve 1) and temperature degradation (curve 2) on the optical density and test temperature of $180^{\circ} \mathrm{C}$ of ALPHA'S $5 \mathrm{~W}-40 \mathrm{SN}$ synthetic motor oil 
2. Предложен показатель интенсивности процессов окисления и температурной деструкции, определяемый приращением скорости этих процессов за установленное время испытания, позволяющий утверждать образование двух видов продуктов различной энергоемкости.

3. Установлено, что продукты окисления повышают противоизносные свойства в 1,2 раза по сравнению с продуктами температурной деструкции.

\section{Список литературы}

[1] Безбородов Ю.Н., Ковальский Б.И., Малышева Н.Н., Сокольников А.Н., Мальцева Е.Г. Методы контроля и диагностики эксплуатационных свойств смазочных материалов по параметрам термоокислительной стабильности и температурной стойкости. Красноярск: СФУ, 2011. 366 c. [Bezborodov Yu.N., Kowalski B.I., Malysheva N.N., Sokolnikov A.N., Maltseva E.G. Methods of monitoring and diagnosing the operational properties of lubricants according to the parameters of thermo-oxidative stability and temperature resistance, Krasnoyarsk: SFU, 2011. 366 p. (in Russian)]

[2] Kovalski B. I., Bezborodov Yu.N., Agrovichenko D.V. Research results of makeup influence on semi-synthetic motor oils thermal oxidation stability, IOP Conference Series: Earth and Environmental Science, 2019, $6 \mathrm{p}$.

[3] Ермилов Е.А., Ковальский Б.И., Безбородов Ю.Н., Балясников В.А. Окисление и температурная деструкция минерального моторного масла ZIC HIFLO 10W-40 SL в процессе эксплуатации. Мир нефтепродуктов. Вестник нефтяных компаний, 6, 2017, $26-29$ [Ermilov E.A., Kovalsky B.I., Bezborodov Yu.N., Balyasnikov V.A. Oxidation and thermal degradation of ZIC HIFLO 10W-40 SL mineral motor oil during operation, The world of petroleum products. Bulletin of Oil Companies, 6, 2017, 26-29 (in Russian)]

[4] Ермилов Е.А., Ковальский Б.И., Безбородов Ю.Н., Балясников В.А. Оценка влияния температуры на процессы окисления и температурной деструкции частично-синтетического моторного масла Castrol Magnatec 10W-40 R SL/CF, Известия ТулГУ, 5, 2017, 99-106 [Ermilov E.A., Kovalsky B.I., Bezborodov Yu.N., Balyasnikov V.A. The assessment of influence of temperature on processes of oxidation and thermal destruction of part synthetic motor oil Castrol Magnatec 10W-40 R SL/CF, News of TulSU, 5, 2017, 99-106 (in Russian)]

[5] Ermilov E.A., Kovalski B.I., Balyasnikov V.A., Agrovichenko D.V., Oleynik V.Z., Afanasov V.I. Substantiation of optical criterions of thermal-oxidative stability of lubricating oil, IOP Conference Series: Earth and Environmental Science, 2019, 8 p.

[6] Besser C., Steinschütz K., Dorr N., Novotny-Farkas F., Allmaier G. Impact of engine oil degradation on wear and corrosion caused by acetic acid evaluated by chassis dynamometer bench tests, Elsevier: Wear, 2014, 317, 64-76.

[7] Lubricant Testing. Focussing on Mechanico-Dynamical tests, Klüber Lubrication München, 2003, 09/03, 5 p.

[8] Useful information in scuffing load tests, Klüber Lubrication München, 2006, 09/06, 7 p. 